\title{
PERFIL DOS ATENDIMENTOS A PACIENTES ONCOLÓGICOS EM UMA UNIDADE DE PRONTO ATENDIMENTO
}

\section{PROFILE OF CARE MEASURES PROVIDED TO CANCER PATIENTS IN AN EMERGENCY CARE UNIT}

\section{PERFIL DE LOS ATENDIMENTOS A PACIENTES ONCOLÓGICOS EN UNA UNIDAD DE PRONTO ATENCIÓN}

Marcela Moysés Gonçalves ${ }^{1}$, Natália de Almeida Barbosa Guedes ${ }^{1}$, Selme Silqueira de Matos ${ }^{2}$, Sabrina Daros Tiensoli ${ }^{3}$, Giovana Paula Resende Simino ${ }^{4}$, Allana dos Reis Corrêa ${ }^{2}$.

\section{RESUMO}

Objetivo: descrever o perfil dos atendimentos realizados a pacientes oncológicos admitidos na unidade de pronto atendimento de um hospital de grande porte. Métodos: estudo descritivo, quantitativo que avaliou 315 prontuários de pacientes oncológicos, com idade maior ou igual a 18 anos, submetidos a classificação de risco em um pronto atendimento. Os dados foram coletados em prontuários eletrônicos e submetidos à análise estatística descritiva. Resultados: predominou o sexo feminino (55,9\%), mediana da idade de 62 anos e o câncer de mama feminino foi o tipo de câncer mais frequente (20,9\%). A queixa principal foi dor (36,2\%), a maioria dos pacientes foi classificada como pouco urgente/verde $(54 \%)$, seguido de urgente/amarelo (41,6\%). A conduta mais frequente foi a administração de medicamentos $(64,1 \%)$ e $70,9 \%$ recebeu alta para o domicílio. Conclusão: observou-se a necessidade de investimentos em tratamentos alternativos para o manejo da dor em pacientes oncológicos e melhorias nos registros dos prontuários visando uma avaliação mais precisa e eficaz dos atendimentos.

Descritores: Oncologia; Serviços médicos de emergência; Emergências; Triagem; Enfermagem.

\section{ABSTRACT}

Objective: to describe the profile of the care provided to cancer patients received in the emergency care unit of a large hospital. Methods: a descriptive, quantitative study evaluated 315 medical records of cancer patients, aged 18 years or older, who were submitted to risk classification in an emergency care unit. Data were collected from electronic charts and submitted to descriptive statistical analysis. Results: Females predominated (55.9\%), with a median age of 62 years, and female breast cancer was the most frequent type of cancer (20.9\%). The main complaint was pain (36.2\%), and the majority was classified as not urgent/green (54.0\%), followed by urgent/yellow (41.6\%). The most frequent treatment was drug administration (64.1\%) and $70.9 \%$ were discharged to go home. Conclusion: it was observed the need for investments in alternative treatments for the management of pain in cancer patients and improvements in the filling of medical records aiming at a more accurate and effective evaluation of the care provided.

Keywords: Oncology; Emergency medical services; Emergencies; Triage; Nursing.

\section{RESUMEN}

Objetivo: describir el perfil de las visitas a los pacientes con cáncer ingresados en la unidad de cuidados de emergencia de un hospital grande. Métodos: Estudio descriptivo que evaluó 315 historias clínicas de pacientes con cáncer, mayores de 18 años, sujeto a la clasificación de riesgo en la atención inmediata. Los datos fueron recolectados en registros electrónico y sometidos al análisis estadístico descriptivo. Resultados: las mujeres (55,9\%), con una edad media de 62 años y el cáncer de mama femenino fueron los más frecuentes $(20,9 \%)$. La queja principal fue el dolor $(36,2 \%)$, la mayoría de los pacientes fue clasificada como no urgente/verde $(54,0 \%)$, seguido por urgente/amarillo $(41,6 \%)$. El método más frecuente fue la administración de medicamentos $(64,1 \%)$ y el $70,9 \%$ recibió el alta para el domicilio. Conclusión: se observó la necesidad de inversiones en tratamientos alternativos para el manejo del dolor en pacientes oncológicos y mejoras en el registro de los prontuarios visando una evaluación más precisa y eficaz de las atenciones.

Descriptores: Oncología; Servicios médicos de urgencia; Urgencias médicas; Triaje; Enfermería.

${ }^{1}$ Graduada em Enfermagem pela Universidade Federal de Minas Gerais. ${ }^{2}$ Graduada em Enfermagem. Doutora em Enfermagem pela Universidade Federal de Minas Gerais. Docente na Universidade Federal de Minas Gerais. ${ }^{3}$ Graduada em Enfermagem. Doutoranda em Enfermagem pela Universidade Federal de Minas Gerais. ${ }^{4}$ Graduada em Enfermagem. Doutora em Medicamentos e Assistência Farmacêutica pela Universidade Federal de Minas Gerais. Docente na Universidade Federal de Minas Gerais.

\section{Como citar este artigo:}

Gonçalves MM, Guedes NAB, Matos SS, et al. Perfil dos Atendimentos a Pacientes Oncológicos em uma Unidade de Pronto Atendimento. Revista de Enfermagem do Centro-Oeste Mineiro. 2018;8:e2595. [Access http://dx.doi.org/10.19175/recom.v7i0.2595 ]; Available in: DOI: 


\section{INTRODUÇÃO}

As estimativas mundiais para 2030, segundo a Organização Mundial da Saúde (OMS), são de 21,4 milhões de novos casos de câncer com 13,2 milhões de mortes, em consequência do crescimento e do envelhecimento da população e, em contrapartida, haverá a redução na mortalidade infantil e nas mortes por doenças infecciosas ${ }^{(1)}$.

Atualmente, o câncer constitui a segunda causa de morte no Brasil, atrás somente das doenças do aparelho circulatório. Estima-se que, gradativamente, será a principal causa de morte no mundo se medidas preventivas não forem implementadas de forma efetiva na população ${ }^{(1-}$ 2). A estimativa para o Brasil, biênio 2016-2017, aponta a ocorrência de cerca de 600 mil novos casos desse agravo. Excluindo-se os casos de câncer de pele, não melanoma, os tipos mais frequentes em homens serão próstata $(28,6 \%)$, pulmão $(8,1 \%)$, intestino $(7,8 \%)$, estômago $(6 \%)$ e cavidade oral $(5,2 \%)$. Nas mulheres, os cânceres de mama $(28,1 \%)$, intestino $(8,6 \%)$, colo do útero $(7,9 \%)$, pulmão $(5,3 \%)$ e estômago $(3,7 \%)$ figurarão entre os principais ${ }^{(2)}$.

Diante desta realidade, torna-se cada vez mais frequente $o$ atendimento a pacientes oncológicos em praticamente todos os serviços de saúde, principalmente nas unidades hospitalares, com sinais e sintomas relacionados, direta ou indiretamente, ao câncer ou ao seu tratamento. Especificamente, no ambiente intrahospitalar, as unidades de alta complexidade têm como finalidade essencial contribuir para a restauração da qualidade de vida deste grupo de pacientes $^{(2-4)}$.

As emergências oncológicas podem ter início súbito ou demorar meses para desenvolverem e manifestam-se abruptamente, resultando em complicações e agravamentos da doença, o que pode acarretar em óbito, necessitando assim de intervenções rápidas e invasivas. Estas situações emergenciais podem ocorrer a qualquer momento e implicam, na maioria das vezes, na busca por atendimento em unidades de urgência e emergência ${ }^{(3-4)}$.

Outros fatores que provavelmente contribuem para que os pacientes oncológicos busquem as unidades de pronto atendimento, para a resolutividade de suas queixas, mesmo que estas não caracterizem situações de urgências e/ou emergências, incluem o receio de não receberem atendimento ambulatorial efetivo e a possibilidade de acesso imediato a exames de baixa complexidade e medicamentos orais ou intravenosos ${ }^{(5)}$.

A carência de estudos que descrevam o paciente oncológico no cenário das unidades destinadas ao atendimento das urgências e emergências mostra uma importante lacuna de conhecimento na literatura nacional, indicando a necessidade de investimento em estudos mais aprofundados sobre esta temática, com a finalidade de conhecer esta realidade e contribuir para a qualidade dos atendimentos a esses pacientes.

É possível que o desenvolvimento de pesquisas que analisem os atendimentos aos pacientes oncológicos, em unidades de urgência e emergência, favoreça a identificação de suas necessidades específicas, e contribuam para a elaboração e implementação de capacitações e condutas que visem ampliar o conhecimento da equipe multiprofissional, qualificar a assistência prestada a esses pacientes e, consequentemente, melhorar sua qualidade de vida.

Assim, considerando a estimativa de aumento do número de pessoas acometidas por câncer e a necessidade de conhecer as circunstâncias que levam os pacientes oncológicos a buscarem assistência imediata em unidades de urgência e emergência, foi proposto este estudo, que teve por objetivo descrever o perfil dos atendimentos realizados a pacientes oncológicos admitidos na unidade de pronto atendimento de um hospital de grande porte.

\section{MÉTODO}

Trata-se de estudo quantitativo, descritivo, retrospectivo, realizado no Pronto Atendimento (PA) de um hospital de grande porte, mantido por uma entidade filantrópica, localizado no município de Belo Horizonte, Minas Gerais. Nessa instituição, a assistência médica integral, ambulatorial e de internação atende às operadoras de saúde suplementar, clientes privados e usuários do Sistema Único de Saúde ${ }^{(6)}$.

O PA atende, em média, 250 pacientes/dia e utiliza a Classificação de Risco para priorização dos atendimentos, sendo esta realizada por enfermeiros e baseada no Sistema de Triagem de Manchester (STM) ${ }^{(6)}$.

A metodologia utilizada pelo STM foi planejada de forma a estabelecer prioridades de 
atendimento, determinando tempo máximo para avaliação do paciente ${ }^{(7)}$. Organiza-se por meio de 55 fluxogramas (guiados pela queixa principal que motiva a procura pelo serviço) e baseia-se nos discriminadores (sinais e sintomas) que indicam o nível de prioridade clínica. As prioridades do protocolo são classificadas de acordo com um número, nome, cor e tempo de resposta máximo em minutos, sendo: 1: Emergência/Vermelho/0 min; 2: Muito Urgente/Laranja/10 min; 3: Urgente/Amarelo/60 min; 4: Pouco Urgente/Verde/120 $\min$ e 5: Não Urgente/Azul/240 $\mathrm{min}^{(7)}$.
Foram incluídos 315 prontuários de pacientes com câncer, atendidos no período de janeiro a fevereiro de 2015, com idade maior ou igual a 18 anos, submetidos a Classificação de Risco e que, primeiramente, receberam atendimento na instituição. Foram excluídos os pacientes com histórico de câncer com tratamento concluído ou em pesquisa do diagnóstico dessa doença. O fluxograma de inclusão e determinação da amostra analisada está apresentado na Figura 1.

Figura 1 - Fluxograma de inclusão e determinação da amostra estudada. Belo Horizonte, 2016.

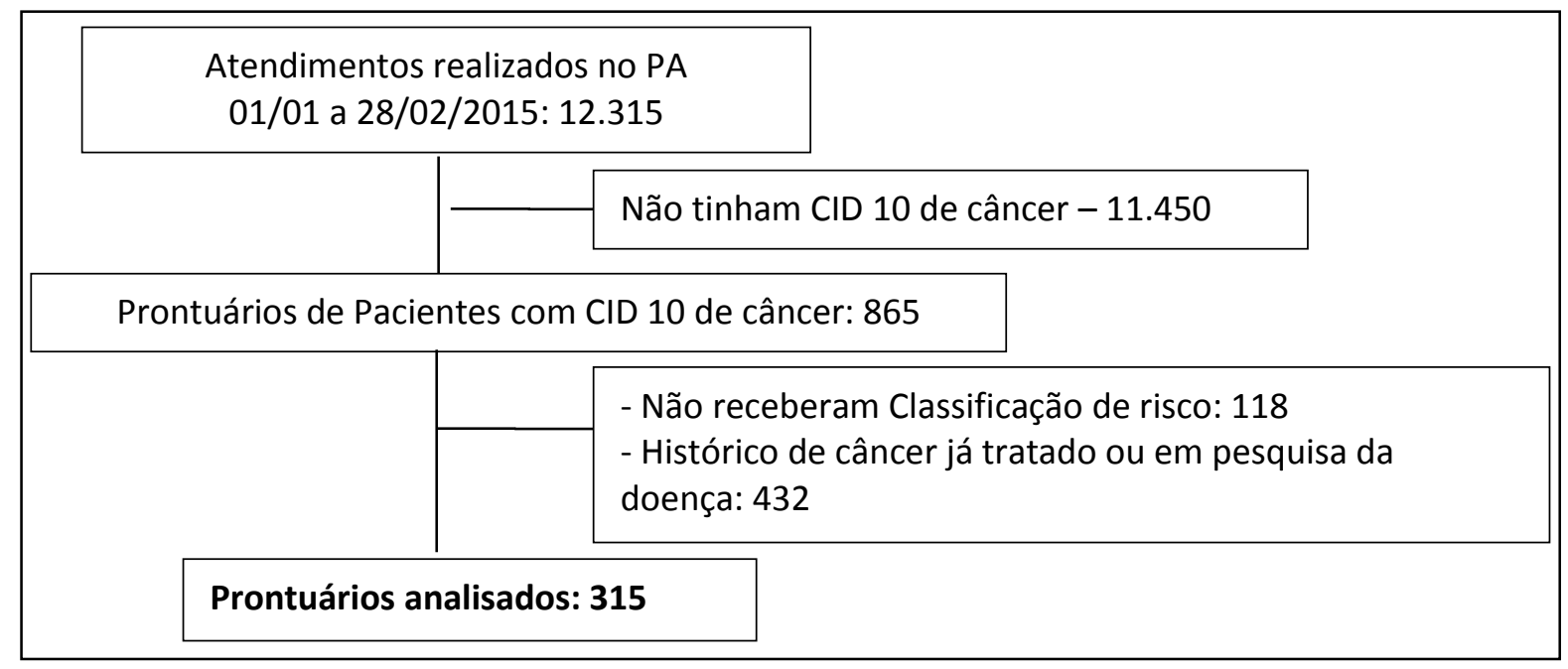

Fonte: Autoria própria

Para caracterização da amostra foram analisadas variáveis sociodemográficas e clínicas da população estudada, sendo: sexo, idade, estado civil, comorbidades, tipo de câncer e terapêutica para o tratamento do câncer. As variáveis relacionadas ao atendimento foram: dia da semana, queixa principal, fluxograma, prioridade de atendimento, conduta terapêutica, exames realizados e desfecho do atendimento.

Foi elaborado, pelas autoras, um instrumento para a coleta dos dados, contemplando as variáveis do estudo. A coleta, realizada pelas autoras, ocorreu nos meses de janeiro a abril de 2016, e foi realizada por meio de consulta aos prontuários eletrônicos, filtrados pelo CID 10, sendo os dados digitados no programa estatístico informatizado Epilnfo (versão 3.5.1).
Para a análise estatística utilizou-se o programa Statistical Software for Professional (Stata), versão 12.0 (Stata Corp., Texas, USA). Na análise descritiva dos dados, a mediana e o intervalo interquartílico (IQ) foram calculados para a variável quantitativa, uma vez que essa não apresentou distribuição normal (teste de Shapiro-Wilk). As frequências e as proporções foram calculadas para as variáveis categóricas.

O projeto de pesquisa, por atender as exigências da Resolução 466/12, do Conselho Nacional de Saúde, foi aprovado pelo Comitê de Ética em Pesquisa da Universidade Federal de Minas Gerais e da instituição coparticipante, parecer $\mathrm{n}$ ㅇ 1.174.638/2015.

\section{RESULTADOS E DISCUSSÃO}


Os dados coletados possibilitaram análises das características sociodemográficas, clínicas e dos atendimentos realizados aos pacientes oncológicos.

Dos 315 prontuários que compuseram a amostra, $55,9 \%$ das pessoas eram do sexo feminino. A idade variou de 22 a 96 anos, com mediana de 62 anos (IQ: 51-74).

Os resultados encontrados nesta pesquisa acompanham o perfil epidemiológico do câncer no Brasil e evidenciam que mais da metade dos pacientes são do sexo feminino. Estudo desenvolvido em município da região sul do Brasil mostrou resultado semelhante ${ }^{(8)}$. A estimativa de novos casos de câncer para 2016, realizada pelo Instituto Nacional do Câncer (INCA), indicava a ocorrência de 300.870 novos diagnósticos de cânceres em mulheres e 295.200 em homens, demonstrando, assim, uma perspectiva de maior incidência do desenvolvimento de câncer em mulheres quando comparada aos homens(2). Sabe-se que, habitualmente, as mulheres procuram os serviços de saúde com maior frequência, aumentando as chances de diagnóstico precoce e tratamento oportuno das doenças $^{(9)}$. Já os homens recorrem ao serviço quando a doença está mais avançada ou frequentemente não aderem ao tratamento ${ }^{(10)}$.

A mediana de idade foi semelhante à encontrada em estudo desenvolvido em município do interior do estado de São Paulo ${ }^{(11)}$. É significativo o aumento da prevalência de câncer com o aumento da idade, uma vez que, com o envelhecimento da população, as doenças crônicas passaram a representar uma expressiva e crescente demanda aos serviços de saúde ${ }^{(9)}$.

Quanto ao estado civil, a maior parcela da amostra estudada era casada (61\%), seguida por solteiros (17,6\%), viúvos (10,5\%) e divorciados/separados (9,9\%). O fato de se viver com companheiro é considerado benéfico e pode influenciar no estado de saúde do portador de neoplasia, uma vez que a presença de um companheiro oportuniza apoio social, reduz os efeitos do estresse e auxilia na manutenção do tratamento e na sobrevida do paciente ${ }^{(12)}$.

Os dados referentes às variáveis comorbidades, tipo de câncer e terapêutica para o tratamento do câncer estão apresentados na Tabela

Tabela 1 - Comorbidades, tipo de câncer e terapêutica para o tratamento do câncer de pacientes oncológicos atendidos no pronto atendimento. Belo Horizonte, 2016.

\begin{tabular}{|c|c|c|}
\hline Variável & $\mathbf{N}$ & $\%$ \\
\hline Comorbidades & 299* & 100 \\
\hline Hipertensão arterial sistêmica & 127 & 42,5 \\
\hline Diabetes Mellitus & 46 & 15,4 \\
\hline Doença cardíaca & 27 & 9 \\
\hline Hipotireoidismo & 24 & 8 \\
\hline Doença respiratória crônica & 21 & 7 \\
\hline Outras & 54 & 18,1 \\
\hline Tipo de câncer & 315 & 100 \\
\hline Mama & 66 & 20,9 \\
\hline Próstata & 44 & 14 \\
\hline Cólon e Reto & 32 & 10,2 \\
\hline Cabeça e pescoço & 23 & 7,3 \\
\hline Rede linfática & 21 & 6,7 \\
\hline Outros & 129 & 40,9 \\
\hline
\end{tabular}




\begin{tabular}{lrr}
\hline Terapêutica para o tratamento do câncer & $\mathbf{4 8 9 * *}$ & $\mathbf{1 0 0}$ \\
Quimioterapia & 179 & 36,6 \\
Intervenção cirúrgica & 176 & 36 \\
Radioterapia & 102 & 20,9 \\
Hormonioterapia & 32 & 6,5
\end{tabular}

* Nem todos os prontuários estavam preenchidos com as variáveis estudadas e um mesmo prontuário poderia apresentar mais de uma comorbidade.

**O paciente pode ter sido submetido a mais de uma opção de terapêutica.

Fonte: Autoria própria

Havia registro de comorbidades em 178 prontuários (56,5\%) e, conforme demonstrado na Tabela 1, um mesmo paciente apresentou mais de uma comorbidade, por isso o valor absoluto foi de 299 comorbidades. A categoria "outras" agrupou as comorbidades com frequência igual ou inferior a 16 na amostra, sendo: doença psiquiátrica $(n=16)$, dislipidemia $(n=14)$, doença renal $(n=7)$, obesidade $(n=6)$, tabagismo $(n=5)$, artrite $(n=1)$, esclerose lateral amiotrófica $(n=1)$, fibromialgia $(n=1)$, hipertireoidismo $(n=1)$, lúpus eritematoso sistêmico $(n=1)$ e síndrome da imunodeficiência adquirida $(n=1)$.

A categoria "outros" da variável tipo de câncer foi composta pelos tipos com frequência inferior a 20 casos, sendo: leucemia $(n=16)$, ovário $(n=10)$, pulmão $(n=10)$, pâncreas $(n=8)$, esôfago $(n=7)$, estômago $(n=7)$, ósseo $(n=7)$, pele $(n=7)$, bexiga $(n=6)$, cerebral $(n=6)$, colo do útero $(n=5)$, intestino $(n=5)$, mieloma múltiplo $(n=5)$, fígado $\quad(n=4), \quad$ rim $\quad(n=4)$, indeterminado/disseminado $(n=3)$, glioblastoma $(n=3)$, língua $(n=3)$, tireóide $(n=3)$, vesícula biliar $(n=3)$, pênis $(n=2)$, coluna $(n=1)$, endométrio $(n=1)$, faringe $(n=1)$, mielofibrose $(n=1)$ e vulva $(n=1)$. Em relação à intervenção cirúrgica, a cirurgia mais realizada foi mastectomia total $(18,7 \%)$, seguida de prostatectomia radical $(10,8 \%)$.

Os tipos de câncer com maior frequência na amostra estudada foram os cânceres de mama, seguidos por próstata, colón/reto e cabeça/pescoço. Estudo desenvolvido na região sul do país evidenciou câncer de mama e próstata como os mais frequentes(8). Sem considerar os tumores de pele não melanoma, o câncer de mama é mundialmente o tipo de câncer mais frequente em mulheres. Para o Brasil, em 2016, eram esperados 57.960 casos novos de câncer de mama, com um risco estimado de 56,20 casos a cada 100 mil mulheres ${ }^{(2)}$. Observa-se este tipo de câncer também como o mais prevalente na amostra estudada. O câncer de mama, possivelmente, é a neoplasia mais temida pelas mulheres, uma vez que a sua ocorrência causa grande impacto psicológico, funcional e social, atuando negativamente nas questões relacionadas à autoimagem e à percepção da sexualidade ${ }^{(13)}$.

$\mathrm{Na}$ população masculina, o câncer de próstata sobressaiu em relação aos demais tipos. A expectativa para o ano de 2016 era que, dos 295.200 novos diagnósticos de casos de câncer em homens, 61.200 fossem de próstata ${ }^{(2)}$. O terceiro câncer mais prevalente na amostra estudada foi o câncer de colón e reto. Estimavase, para 2016, no Brasil, 16.660 casos novos deste tipo de câncer em homens e 17.620 em mulheres, correspondendo à terceira neoplasia mais incidente na população masculina e a segunda na população feminina no Brasil, excluindo-se os tumores de pele ${ }^{(2)}$. É provável que o investimento em estratégias que incentivem o rastreamento do câncer de colón e reto, assim como ocorre para os cânceres de próstata, colo uterino e, principalmente o de mama, favoreça a identificação de lesões em estágios iniciais, menos sintomáticas e com maior chance de cura, podendo assim contribuir para a redução da incidência, mortalidade e gastos públicos relacionados a esse tipo de câncer ${ }^{(14)}$.

Os cânceres de cabeça e pescoço representaram o quarto tipo mais frequente nos pacientes que procuraram atendimento no pronto socorro. Em 2014, a estimativa para a ocorrência de câncer de cavidade oral no Brasil era de 11.280 casos novos em homens e 4.010 em mulheres ${ }^{(1)}$. O tratamento desse tipo de câncer implica na realização de cirurgias que envolvem grandes ressecções, com possibilidade de ocorrer mutilações funcionais e estéticas, que 
repercutem na vida diária dos pacientes. Além da abordagem cirúrgica, a necessidade de radioterapia pode contribuir para o surgimento de complicações clínicas como mucosites e dermatites $^{(15)}$. Esses podem ser fatores que contribuíram para a procura de atendimento em unidades de urgência e emergência.

As terapêuticas realizadas com maior frequência nos pacientes deste estudo foram quimioterapia e intervenção cirúrgica. As intervenções cirúrgicas, assim como a quimioterapia antineoplásica, tem-se tornado uma das mais importantes e promissoras maneiras de combater o câncer e podem ser empregadas com finalidade curativa ${ }^{(16)}$. Os objetivos dos tratamentos do câncer são a cura, aumentar a sobrevida quando não há possibilidade de cura e proporcionar cuidados paliativos. Quando o tratamento não pode resultar em cura, ele deve levar a uma melhora do bem-estar e da qualidade de vida dos pacientes $^{(16)}$.

O dia da semana com maior frequência de atendimentos foi segunda-feira $(22,9 \%)$, seguido da sexta-feira $(20,6 \%)$, no período matutino. A associação entre a adequação da demanda e horário da procura, com maior proporção de casos com pouca urgência, no horário diurno, corroboram os dados encontrados em outros dois estudos desenvolvidos no $\mathrm{Brasil}^{(8-9)}$.

As queixas mais frequentes foram dor $(36,2 \%)$ e fraqueza $(6,7 \%)$, seguidas de outras com frequência inferior a 20 relatos: lesão cutânea e abscessos ( $n=19)$, febre $(n=17)$, dispneia $(n=14)$, cansaço e mal estar $(n=12)$, vômito $(n=12)$, disúria $(n=9)$, edema $(n=8)$, anúria $(n=7)$, diarreia $(n=7)$, tontura $(n=7)$, avaliação de especialista $(n=5)$, constipação $(n=5)$, distenção abdominal $(n=4)$, retirada de pontos $(n=4)$, hematúria, sangramento anal ou melena $(n=3)$, hemotransfusão $(n=3)$, obstrução de ouvido $(n=3)$, prurido $(n=3)$, secreção local $(n=3)$, trauma $(n=3)$, desmaio $(n=2)$, desvio de comissura labial $(n=2)$, epistaxe $(n=2)$, exame laboratorial alterado $(n=2)$, ferida $(n=2)$, realização de quimioterapia $(n=2)$, retirada de dreno $(n=2)$, retirada de sonda nasogástrica $(n=2)$, retirada/perda de sonda vesical de demora $(n=2)$, tosse $(n=2)$, vazamento de sonda $(n=2)$, desidratação $(n=1)$, disfagia $(n=1)$, equimose $(n=1)$, movimentos involuntários $(n=1)$, náuseas $(n=1)$, perda de consciência $(n=1)$, perda de visão $(n=1)$, prostração $(n=1)$ e trombose venosa profunda $(n=1)$.

A alta prevalência de dor foi evidenciada em estudos que analisaram o perfil dos atendimentos gerais em unidades de Pronto Atendimento ${ }^{(6,11,17)}$. Dados da Organização Mundial de Saúde (OMS) mostram que, das cinco milhões de pessoas que morrem de câncer por ano, $80 \%$ não têm um controle devido da dor. No Brasil, a estimativa é que, de 62 a $90 \%$ dos pacientes oncológicos apresentam algum tipo de dor, sendo o segundo país da América Latina em que os portadores de câncer mais sentem dor ${ }^{(18)}$.

Destaca-se a importância de investimentos em capacitação dos enfermeiros na avaliação adequada da dor quanto à localização, intensidade, duração e tratamentos disponíveis, bem como a necessidade da utilização de escalas apropriadas ao público atendido, padronização desta avaliação, objetivando reduzir os aspectos subjetivos que podem modificar o nível de dor atribuído ao paciente ${ }^{(17)}$.

Dos 52 fluxogramas de apresentação que compunham o STM à época do estudo, 22 $(42,3 \%)$ foram referidos na Classificação de Risco. Os fluxogramas citados com frequência igual ou superior a 10 estão apresentados na Figura 2.

Figura 2 - Distribuição dos fluxogramas de apresentação mais frequente na classificação de risco dos pacientes oncológicos. Belo Horizonte, 2016.

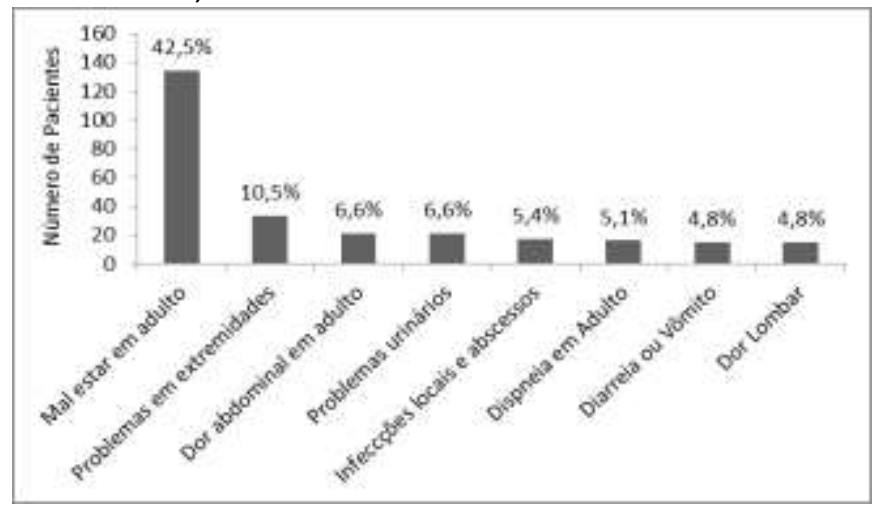

Fonte: Autoria própria. 
Os fluxogramas com frequência inferior a 10 casos representaram $63,6 \%$ da amostra e não apareceram na figura acima (Figura 2), sendo: feridas ( $n=9)$, problemas em ouvidos $(n=7)$, dor de garganta $(n=5)$, dor torácica $(n=5)$, alergia $(n=3)$, problemas em face $(n=3)$, cefaleia $(n=2)$, hemorragia digestiva $(n=2)$, traumatismo craniano $(n=2)$, alteração de comportamento $(n=1)$, desmaio no adulto $(n=1)$, história de diabetes $(n=1)$, problemas em olhos $(n=1)$ e traumatismo torácico e abdominal $(n=1)$.

Estudo realizado em serviço de emergência de grande porte da região Sul do Brasil, que utiliza o STM para classificação de risco, mostrou resultado semelhante, sendo o fluxograma "Malestar em adulto", o mais citado em pacientes classificados como não urgentes (azul), pouco urgentes (verde) e urgentes (amarelo) ${ }^{(19)}$. Ressalta-se que, mesmo a dor sendo referida como queixa principal mais citada pelos pacientes e que o STM possui fluxogramas referentes a diversos tipos de dor, como dor abdominal, cefaleia, dor lombar, esses não aparecem como os mais citados, o que reporta à possibilidade de uma subnotificação da dor como foco principal do atendimento.

Quanto à prioridade de atendimento determinada pela classificação de risco, a maioria foi classificada como prioridade 4: pouco urgente/verde (54\%), seguida de 3: urgente/amarela $(41,6 \%)$ e 2 : muito urgente/laranja $(4,4 \%)$. Observa-se que mais da metade dos pacientes não demandava atendimento imediato. Essa realidade é determinante para a superlotação do serviço, fenômeno mundial que se caracteriza pela ocupação dos leitos de emergência, internação em corredores, aumento do tempo de espera para atendimento, desgaste da equipe assistencial e grande pressão para novos atendimentos ${ }^{(20)}$. Estima-se que muitas dessas situações possam ter sido decorrentes de doenças crônicas não tratadas e que tenham agudizado pela falta ou limitações da assistência na Rede de Atenção Básica à Saúde ${ }^{(11,20)}$.

Os dados referentes à conduta terapêutica, exames realizados e desfecho dos atendimentos estão apresentados na Tabela 2.

Tabela 2- Conduta terapêutica, exames realizados e desfecho dos atendimentos realizados a pacientes oncológicos no pronto atendimento. Belo Horizonte, 2016.

\begin{tabular}{|c|c|c|}
\hline Variável & $\mathbf{N}$ & $\%$ \\
\hline Conduta terapêutica & $348^{*}$ & 100 \\
\hline Medicação via oral & 138 & 39,7 \\
\hline Medicação parenteral & 77 & 22,2 \\
\hline Soroterapia & 46 & 13,2 \\
\hline Medicação tópica & 21 & 6 \\
\hline Hemotransfusão & 21 & 6 \\
\hline Outras & 45 & 12,9 \\
\hline Exames realizados & $298 * *$ & 100 \\
\hline Exames de imagem & 108 & 36,3 \\
\hline Hemograma & 61 & 20,5 \\
\hline Revisão laboratorial & 60 & 20,1 \\
\hline Exame de Urina rotina & 23 & 7,7 \\
\hline Outros & 46 & 15,4 \\
\hline Desfecho & $313 * *$ & 100 \\
\hline
\end{tabular}


Alta para domicílio

Internação
222

70,9

$91 \quad 29,1$

* Os pacientes foram submetidos a mais de uma conduta terapêutica.

**Nem todos os prontuários foram preenchidos com a variável.

Fonte: Autoria própria.

Em relação às condutas terapêuticas a categoria "outras" agrupou as condutas que tiveram frequência inferior a 12 registros, sendo: cateterismo vesical de alívio e demora $(n=10)$, curativo de feridas e imobilização com tipoia $(n=10)$, medicação subcutânea $(n=6)$, medicação inalatória $(n=4)$, retirada de pontos $(n=4)$, suturas ou retirada de corpo estranho $(n=4)$, sondagem gástrica $(n=3)$, retirada de cateter vesical $(n=2)$, sondagem entérica $(n=2)$ e retirada de dreno torácico $(n=1)$. Cabe ressaltar que o mesmo paciente pode ter recebido uma ou mais condutas, simultaneamente.

Os exames realizados que compuseram a categoria "outros" tiveram frequência igual ou inferior a 16 registros, sendo: Gram de gota $(n=16)$, urocultura $(n=15)$, eletrocardiograma $(n=10)$, ionograma ( $n=4)$ e função hepática $(n=1)$.

A prescrição de medicamentos, sob a forma de principal conduta, reforça o modelo de atenção baseado na queixa-conduta dos casos agudos, buscando resolver as necessidades de saúde trazidas pelos usuários ${ }^{(5)}$. Portanto, são condutas de resolução em curto prazo, fato que explica os retornos subsequentes dos mesmos usuários ao pronto atendimento, ou seja, quando retornam os sintomas, novamente, os usuários procuram atendimento. Dessa forma, entende-se que o PA ainda se constitui em serviço que possui um grande acúmulo de possibilidades de oferta de serviços a serem utilizados pelos usuários, seja na forma de medicamentos, exames e/ou consultas ${ }^{(5)}$.

A administração de medicação oral ou endovenosa foi conduta para alívio de sintomas em mais da metade dos pacientes avaliados neste estudo. Esse dado pode estar relacionado ao fato de a dor ter sido a principal queixa reportada. Estudos apontam que a prescrição de medicamentos sintomáticos por via oral ou endovenosa como conduta terapêutica fica em evidência dentre as condutas médicas instituídas e que, em sua maioria, estão diretamente relacionadas à resolução das queixas relatadas pelos usuários ${ }^{(5,11)}$.

Em relação ao desfecho, a maior frequência de alta para domicílio foi semelhante ao evidenciado num estudo brasileiro ${ }^{(8)}$. Esse resultado nos faz questionar se os pacientes oncológicos que procuram os serviços de emergência poderiam resolver demandas com menor grau de urgência em consultas de rotina ou com o acompanhamento ambulatorial. Acredita-se que, muitos pacientes procuraram os serviços de urgência e emergência para agilizar o acesso imediato a medicamentos não prescritos nas consultas de rotina ou até mesmo por não receberem o cuidado adequado em serviços de atenção primária $^{(8)}$.

No que diz respeito às limitações do estudo, deve-se considerar que se trata de dados secundários, em que pode ter ocorrido o preenchimento incompleto dos prontuários. Destaca-se ainda que a instituição pesquisada não utiliza o sistema eletrônico formal do STM, o que limitou a busca de dados essenciais como tempo de espera para a classificação, tempo de duração da classificação de risco e tempo entre classificação e o primeiro atendimento.

\section{CONCLUSÃO}

Este estudo traçou o perfil clínico, sociodemográfico e de atendimentos dos pacientes oncológicos que procuraram um pronto atendimento de um hospital de grande porte. A maioria dos pacientes era mulher, com mediana da idade de 62 anos, casada, com diagnóstico de câncer de mama e predomínio da realização de quimioterapia como tratamento para o câncer.

Os atendimentos ocorreram com maior frequência às segundas-feiras, no período diurno. A queixa principal foi dor, o fluxograma mais citado foi "mal-estar em adulto" e a maioria foi classificada como não urgente/verde. A conduta mais frequente foi a administração de medicamentos por via oral e endovenosa e a maioria recebeu alta do serviço de emergência após melhora dos sintomas.

Por meio desse trabalho, foi possível observar que tratamentos alternativos para o manejo da dor precisam ser estabelecidos para os pacientes oncológicos, uma vez que esse sintoma aparece como queixa principal. Além disso, a 
Rede de Atenção Básica precisa ser fortalecida para atender e acompanhar o tratamento e evolução dos pacientes oncológicos. Observa-se, ainda, a necessidade de melhoria quanto ao registro completo dos prontuários para que se tenha uma avaliação mais precisa e eficaz dos atendimentos realizados a esses pacientes.

\section{REFERÊNCIAS}

1. Instituto Nacional de Câncer José Alencar Gomes da Silva. Estimativa 2014: Incidência de câncer no Brasil. Rio de Janeiro: INCA; 2014.

2. Instituto Nacional de Câncer José Alencar Gomes da Silva Estimativa 2016: Incidência de câncer no Brasil. Rio de Janeiro: INCA; 2016.

3. Gabriel J. Acute oncological emergencies. Nurs Stand. 2012 Oct;27(4):35-41. DOI: 10.7748/ns2012.09.27.4.35.c9308

4. Sadik M, Ozlem K, Huseyin M, AliAyberk B, Ahmet $\mathrm{S}$, Ozgur $\mathrm{O}$. Attributes of cancer patients admitted to the emergency department in one year. World J Emerg Med. 2014;5(2):85-90. DOI: 10.5847/wjem.j.issn.1920-8642.2014.02.001

5. Gomide MFS, Pinto IC, Gomide DMP, Zacharias FCM. Perfil de usuários em um serviço de pronto atendimento. Rev Medicina 2012;45(1): 31-8. DOI: $10.11606 /$ issn.2176-7262.v45i1p31-38

7. Grupo Brasileiro de Classificação de Risco (GBCR). Sistema Manchester de Classificação de Risco: Classificação de risco na urgência e emergência. Oxford: GBCR; 2016.

8. Borges G, Rovere RK, Maman KAS, Zabel MCJ, Dagnoni C, Corrêa EG et al. Perfil dos pacientes oncológicos que procuraram o departamento de emergência de um hospital de Blumenau no período de 01 abril de 2011 a 31 de outubro de 2011. Rev Bras Oncol Clín. 2013;9(34):130-4. Disponível em: http://sboc.org.br/revistasboc/pdfs/34/artigo1.p $\underline{\mathrm{df}}$

9. Oliveira MM, Malta DC, Guauche H, Moura L, Silva GA. Estimativa de pessoas com diagnóstico de câncer no Brasil: Dados da Pesquisa Nacional de Saúde. Rev Bras Epidemiol. 2015 dez;18(suppl 2):146-57. DOI: 10.1590/1980-

\section{$\underline{5497201500060013}$}

10. Tomberg JO, Cantarelli KJ, Guanilo MEE, Dal Pai D. Acolhimento com avaliação e classificação de risco no Pronto Socorro: Caracterização dos atendimentos. Rev Cienc Cuid Saude 2013;12(1):80-7. DOI: $10.4025 /$ cienccuidsaude.v12i1.18694

11. Boaventura AP, Vedovato CA, Santos FF. Perfil dos pacientes oncológicos atendidos em uma unidade de emergência. Rev Cienc Enferm. 2015 ago;21(2):51-62. DOI: $10.4067 /$ S0717$\underline{95532015000200006}$

12. Müller JS, Sperandio FF. Perfil demográfico e fatores associados de pacientes com câncer de mama em tratamento radioterápico na grande Florianópolis. Rev Cienc Med Biol 2012 jan/abr;11(1):41-7. DOI: 10.9771/cmbio.v11i1.5792

13. Pinheiro AB, Lauter DS, Medeiros GC, Cardozo $I R$, Menezes LM; Souza RMB, et al. Câncer de mama em mulheres jovens: Análise de 12.689 casos. Rev Bras Cancerol. 2013;59(3):351-9. Disponível em: http://www1.inca.gov.br/rbc/n 59/v03/pdf/05artigo-cancer-mama-mulheres-jovens-analisecasos.pdf

14. Valadão $M$, Leal RA, Barbosa LC, Carneiro $M$, Muharre RJ. Perfil dos pacientes portadores de câncer colorretal operados em um Hospital Geral: Necessitamos de um programa de rastreamento acessível e efetivo. Rev Bras Coloproct. 2010 abri/jun;30(2):160-6. DOI: 10.1590/S0101$\underline{98802010000200006}$

15. Paula JM, Sawada NO. Qualidade de vida relacionada à saúde de pacientes com câncer em tratamento radioterápico. Rev Rene 2015 jan/fev;16(1):106-13. Disponível em: http://www.revistarene.ufc.br/revista/index.php/ revista/article/view/1868/pdf

16. Andrade V, Sawada NO, Barichello E. Quality of life in hematologic oncology patients undergoing chemotherapy. Rev Esc Enferm USP 2013 Apr;47(2):355-61. DOI: 10.1590/S0080$\underline{62342013000200012}$

17. Diniz AS, Silva AP, Souza CC, Chianca TCM. Clinical demand in an emergency care unit according to the Manchester triage system. Rev Eletrônica Enf. 2014 abr/jun;16(2): 312-20. DOI: 10.5216/ree.v16i2.21587

18. Cunha FF, Rêgo LP. Enfermagem diante da dor oncológica. Rev Dor 2015 abr/jun;16(2): 142-5. DOI: $10.5935 / 1806-0013.20150027$

19. Tanccini T. Sistema de Manchester: Tempo despendido na classificação de risco, prioridades estabelecidas e desfecho clínico dos pacientes atendidos na maior emergência do sul do Brasil [dissertação] Porto Alegre (RS): Escola de Enfermagem da Universidade Federal do Rio Grande do Sul; 2014.

20. Bittencourt RJ, Hortale VA. Interventions to solve overcrowding in hospital emergency services: A systematic review. Cad Saúde Pública 
2009 jul;25(7):1439-54. DOI: 10.1590/S0102-

$\underline{311 \times 2009000700002}$

Nota: Não houve financiamento. Extraído do Trabalho de Conclusão de Curso de Graduação em Enfermagem da Universidade Federal de Minas Gerais - UFMG

Recebido em: 31/10/2017

Aprovado em: 27/04/2018

Endereço de correspondência:

Allana dos Reis Corrêa

Av. Alfredo Balena, 190 - Santa Efigênia

CEP: 30-130 100 - Belo Horizonte/MG - Brasil

E- mail: allanareiscorrea@gmail.com 